\title{
Global dynamics of a viral infection model with a latent period and Beddington-DeAngelis response
}

\author{
Yukihiko Nakata ${ }^{a, b}$ \\ ${ }^{a}$ Department of Pure and Applied Mathematics, Waseda University, 3-4-1 Okubo, Shinjuku-ku, Tokyo, 169-8555, Japan \\ ${ }^{\mathrm{b}}$ BCAM - Basque Center for Applied Mathematics Bizkaia Technology Park, Building 500, E-48160, Derio, Basque \\ Country, Spain
}

\begin{abstract}
In this paper, we study the global dynamics of a viral infection model with a latent period. The model has a nonlinear function which denotes the incidence rate of the virus infection in vivo. The basic reproduction number of the virus is identified and it is shown that the uninfected equilibrium is globally asymptotically stable if the basic reproduction number is equal to or less than unity. Moreover, the virus and infected cells eventually persist and there exists a unique infected equilibrium which is globally asymptotically stable if the basic reproduction number is greater than unity. The basic reproduction number determines the equilibrium that is globally asymptotically stable, even if there is a time delay in the infection.
\end{abstract}

Key words: virus dynamics models; nonlinear incidence rate; global asymptotic stability; uniformly persistence;

\section{Introduction}

To develop a better understanding of a virus dynamics in vivo, mathematical models have played a significant role. By modeling the dynamics of the virus and target cells, much knowledge about the mechanism of the interactions among these components have been gained (see $[1,3-9$, $11-16,18,19]$ and the references therein). First of all, we introduce the standard viral infection model.

$$
\left\{\begin{array}{l}
\frac{d}{d t} x(t)=s-d x(t)-k x(t) v(t), \\
\frac{d}{d t} y(t)=k x(t) v(t)-\delta y(t), \\
\frac{d}{d t} v(t)=N \delta y(t)-\mu v(t) .
\end{array}\right.
$$

Email address: yunayuna.na@gmail.com, nakata@bcamath.org (Yukihiko Nakata).

Preprint submitted to Elsevier

December 10, 2010 
$x(t), y(t)$ and $v(t)$ denote the concentration of uninfected cells, infected cells and free virus particles, respectively. It is assumed that new target cells are generated at a constant $s$ and die at rate $d x(t)$. Infection of target cells by free virus is assumed to occur at rate $k x(t) v(t)$ and die at rate $\delta y(t)$. The average number of virus particles produced over the lifetime of a single infected cell is $N$, which also called the burst size. Hence, new virus are produced from infected cells at rate $N \delta y(t)$ and die at rate $\mu v(t)$. (1.1) has been used to model a virus dynamics in vivo such as human immunodeficiency virus type I (HIV-1) and hepatitis B virus (HBV) (see also [1,3,12-15] and the references therein).

(1.2) has always an uninfected equilibrium $\left(x_{0}, 0,0\right), x_{0}=\frac{s}{d}$, corresponding to the extinction of the infected cells and virus. It is also possible that an infected (internal) equilibrium $\left(\bar{x}_{1}, \bar{y}_{1}, \bar{z}_{1}\right)$ exists. Existence of the infected equilibrium is determined by a parameter which is called the basic reproduction number. The basic reproduction number denotes the average number of newly infected cells generated from one infected cells at the beginning of the infectious process and, for system (1.1), it is given by

$$
R_{0(1.1)}=\frac{k x_{0} N}{\mu},
$$

Korobeinikov [5] established the global dynamics of (1.1) by a method of Lyapunov functional. It is shown that the uninfected equilibrium is globally asymptotically stable if $R_{0(1.1)} \leq 1$, while the infected equilibrium exists and is globally asymptotically stable if $R_{0(1.1)}>1$.

(1.1) is formulated under an assumption that the infection could occur and virus are produced from infected cells instantaneously, once uninfected cells are contacted by the virus particles. However, it has been reported that HIV-1 infection models with intracellular delays change the estimation of kinetic parameters when compared to model without delay such as (1.1) by many authors (see also [3,11,12]). Delayed viral infection models which are described by delay differential equations have been introduced and analyzed (see also [3, 8, 11, 19] and the references therein). Li and Shu [9] studied a viral infection model with delay and bilinear incidence rate and established its global dynamics.

On the other hand, functional form of the incidence rate of the infection have a crucial role for modeling of the virus dynamics. Korobeinikov [7] assumed that the incidence rate is given by an unspecified function of the number of the uninfected cells and virus particles. Li and Ma [8] proposed a delayed HIV-1 infection model with a saturated incidence rate, $k x \frac{v}{1+v}$, instead of the bilinear incidence rate $k x v$. Recently, Huang et al. [4] formulated the following virus dynamics model with Beddington-DeAngelis infection rate

$$
\left\{\begin{array}{l}
\frac{d}{d t} x(t)=s-d x(t)-f(x(t), v(t)), \\
\frac{d}{d t} y(t)=f(x(t), v(t))-\delta y(t), \\
\frac{d}{d t} v(t)=N \delta y(t)-\mu v(t),
\end{array}\right.
$$

with

$$
f(x, v)=\frac{k x v}{1+a x+b v}, a \geq 0, b \geq 0, \text { for }(x, v) \in \mathbb{R}^{2} .
$$

They identified the basic reproduction number for (1.2) as follows

$$
R_{0(1.2)}=\frac{k x_{0} N}{\left(1+a x_{0}\right) \mu}
$$

and established the global dynamics of (1.2) by using two Lyapunov functions which are motivated by Korobeinikov [5,6]. (1.2) also has two possible equilibrium, similar to (1.1), uninfected 
equilibrium $\left(x_{0}, 0,0\right)$ and the infected (internal) equilibrium $\left(\bar{x}_{2}, \bar{y}_{2}, \bar{v}_{2}\right)$. It is shown that the uninfected equilibrium is globally asymptotically stable if $R_{0(1.2)} \leq 1$, while the infected equilibrium exists and is globally asymptotically stable if $R_{0(1.2)}>1$.

It is needed to investigate the global asymptotic stability of the equilibrium, because it helps us to understand the global dynamics, not only local dynamics, of the model. In this paper, to account for the effect of a latent period for the cell infection in (1.2), we assume that virus production occurs after the virus entry with a constant time lag $\tau>0$. The recruitment of infected cells at time $t$ is given by $f(x(t-\tau), v(t-\tau)) \mathrm{e}^{-m \tau}$ where $\mathrm{e}^{-m \tau}$ is the probability of surviving from $t-\tau$ to $t$ and $m$ is a constant death rate for infected cells (but, not yet virus producing cells). Then, we obtain the following viral infection model with a latent period $\tau>0$ and BeddingtonDeAngelis infection rate.

$$
\left\{\begin{array}{l}
\frac{d}{d t} x(t)=s-d x(t)-f(x(t), v(t)), \\
\frac{d}{d t} y(t)=f(x(t-\tau), v(t-\tau)) \mathrm{e}^{-m \tau}-\delta y(t), \\
\frac{d}{d t} v(t)=N \delta y(t)-\mu v(t),
\end{array}\right.
$$

with

$$
f(x, v)=\frac{k x v}{1+a x+b v}, a \geq 0, b \geq 0, \text { for }(x, v) \in \mathbb{R}^{2} .
$$

Obviously, (1.1) and (1.2) can be seen as a special case of (1.3)-(1.4), respectively. (1.3)-(1.4) also includes an HIV-1 infection model which was studied by Li and Ma [8].

In this paper, we investigate the global dynamics of (1.3)-(1.4) by employing a method of Lyapunov functional. The Lyapunov functional is motivated by the works of McCluskey [10], Korobeinikov [5] and Huang et al. [4]. We identify the basic reproduction number $R_{0}$ for (1.3)(1.4) (see Section 2) and it is shown that the uninfected equilibrium is globally asymptotically stable if $R_{0} \leq 1$. On the other hand, (1.3)-(1.4) admits a unique infected equilibrium which is globally asymptotically stable if $R_{0}>1$. Thus, the global dynamics of (1.3)-(1.4) is fully determined by the basic reproduction number $R_{0}$. Moreover, our result derive an affirmative answer for a conjecture by Li and Ma [8]. By applying our result, it is shown that the infected equilibrium of their model is also globally asymptotically stable whenever it exists.

The paper is organized as follows. In Section 2, we introduce some basic results for (1.3)(1.4). Explicit form of the basic reproduction number for (1.3)-(1.4) is given. The existence of a unique infected equilibrium is also discussed. In Section 3, we study the global asymptotic stability of the uninfected equilibrium by using a Lyapunov functional which is motivated by Huang et al. [4]. In Section 4, we show the uniformly persistence of the solution by persistence theory. From our result, the virus and infected cells eventually persist for $R_{0}>1$. Moreover, in Section 5, we show that the infected equilibrium is globally asymptotically stable if $R_{0}>1$ by employing a Lyapunov functional. Lemma 5.1 is a key result to obtain this result. In Section 6, we introduce two viral infection models as examples. We obtain an affirmative answer for the conjecture by Li and Ma [8]. Finally, we offer a brief discussion in Section 7.

\section{Preliminary results}

To investigate the dynamics of (1.3)-(1.4), we set a suitable phase space. For $\tau>0$, we denote by $C=C([-\tau, 0], \mathbb{R})$ the Banach space of continuous functions mapping the interval $[-\tau, 0]$ into 
$\mathbb{R}$ equipped with the sup-norm. The nonnegative cone of $C$ is defined as $C_{+}=C\left([-\tau, 0], \mathbb{R}_{+}\right)$. From the biological meanings, the initial conditions for (1.3)-(1.4) is

$$
x(\theta)=\varphi_{1}(\theta), y(\theta)=\varphi_{2}(\theta), v(\theta)=\varphi_{3}(\theta), \theta \in[-\tau, 0],
$$

where $\varphi_{i}(\theta) \in C_{+}, i=1,2,3$.

Lemma 2.1 All solutions of (1.3)-(1.4) with (2.1) are positive for $t \geq \tau$ if one of the following holds.

i) there exists $\bar{\theta} \in[-\tau, 0)$ such that $\varphi_{1}(\bar{\theta}) \varphi_{3}(\bar{\theta})>0$.

ii) $\varphi_{3}(0)>0$.

iii) $\varphi_{2}(0)>0$.

Furthermore, all solutions are bounded by some positive constant for sufficiently large $t$.

PROOF. The solution $(x(t), y(t), v(t))$ of (1.3)-(1.4) with (2.1) exists and is unique on its maximal interval of existence $[0, \sigma)$ for some $\sigma>0$. We see that $x(t)>0$ for all $t \in(0, \sigma)$. Indeed, this follows from that $\frac{d}{d t} x(t)=s>0$ for any $t \in[0, \sigma)$ when $x(t)=0$ by the first equation of (1.3)-(1.4).

Let us show the positivity of $y(t)$ and $v(t)$. Firstly, we claim that if there exists $t_{1} \geq 0$ such that $y\left(t_{1}\right)>0$ or $v\left(t_{1}\right)>0$ then $y(t)>0$ and $v(t)>0$ for any $t>t_{1}$.

Assume $y\left(t_{1}\right)>0$. Then we have that $y(t)>0$ for $t \in\left(t_{1}, t_{1}+\varepsilon\right)$ where $\varepsilon>0$ is sufficiently small. Suppose that there exists a $t_{2}$ such that $y\left(t_{2}\right)=0$ and $y(t)>0$ for $t \in\left(t_{1}, t_{2}\right)$. We have

with

$$
y\left(t_{2}\right)=\left(y\left(t_{1}\right)+\int_{t_{1}}^{t_{2}} f(x(s-\tau), v(s-\tau)) \mathrm{e}^{-m \tau} \mathrm{e}^{\delta s} d s\right) \mathrm{e}^{-\delta t_{2}},
$$

$$
v(s-\tau)= \begin{cases}\varphi_{3}(s-\tau), & \text { for } s-\tau \leq 0 \\ \left(\varphi_{3}(0)+N \delta \int_{0}^{s-\tau} y(u) \mathrm{e}^{\mu(s-\tau)} d u\right) \mathrm{e}^{-\mu(s-\tau)}, & \text { for } s-\tau>0\end{cases}
$$

It then follows $v(s-\tau) \geq 0$ for $s \in\left[t_{1}, t_{2}\right]$. Then we see $y\left(t_{2}\right) \geq y\left(t_{1}\right) \mathrm{e}^{-\delta t_{2}}>0$ and this leads a contradiction. Moreover, from the above discussion, we obtain

$$
v(t)=\left(\varphi_{3}(0)+N \delta \int_{0}^{t} y(s) \mathrm{e}^{\mu s} d s\right) \mathrm{e}^{-\mu t}>0, t>t_{1} .
$$

Next, assume $v\left(t_{1}\right)>0$. Similar to the above discussion, we also obtain $y(t)>0, v(t)>0$ for $t>t_{1}$.

Now, it is sufficient to show the existence of such $t_{1} \geq 0$. For the case ii) and iii), we can choose $t_{1}=0$. Consider the case i). If $y(\bar{\theta}+\tau)=0$ then we have

$$
\left.\frac{d}{d t} y(t)\right|_{t=\bar{\theta}+\tau}=f\left(\varphi_{1}(\bar{\theta}), \varphi_{3}(\bar{\theta})\right) \mathrm{e}^{-m \tau}-\delta y(\bar{\theta}+\tau)>0,
$$

and hence, $y(t)>0$ for $t \in(\bar{\theta}+\tau, \bar{\theta}+\tau+\varepsilon)$ where $\varepsilon>0$ is sufficiently small. Consequently, for any cases, it follows that $x(t)>0, y(t)>0$ and $v(t)>0$ for any $t \in\left[t_{1}, \sigma\right)$ where $t_{1} \geq \tau$.

For $G(t)=x(t-\tau)+y(t) \mathrm{e}^{m \tau}+\frac{1}{N} v(t)$, it then follows

$$
\frac{d}{d t} G(t)=s-d x(t-\tau)-\delta \mathrm{e}^{m \tau} y(t)-\frac{\mu}{N} v(t) \leq s-\min \{d, \delta, \mu\} G(t),
$$

which implies that $(x(t), y(t), v(t))$ is ultimately bounded on $[0, \sigma)$. Finally, it follows that $(x(t), y(t), v(t))$ exists and is unique and positive for any $t \geq \tau$. 
Lemma $2.2 y(t)$ and $v(t)$ of (1.3)-(1.4) with (2.1) are identically zero for $t>0$ if $\varphi_{1}(\theta) \varphi_{3}(\theta)=$ $\varphi_{2}(0)=\varphi_{3}(0)=0$ for all

PROOF. From the initial conditions, it is clear that $y(t)=0$ for $t \in(0, \tau)$. It then follows $v(t)=0$ for all $t \in(0, \tau)$.

Now, we show $y(t)=v(t)=0$ for $t \in[\tau,+\infty)$. Suppose there exists a $t_{1} \geq \tau$ such that $\frac{d}{d t} y\left(t_{1}\right)>$ 0 and $y\left(t_{1}\right)=0$. By (1.3), we see

$$
f\left(x\left(t_{1}-\tau\right), v\left(t_{1}-\tau\right)\right) \mathrm{e}^{-m \tau}>0,
$$

which implies $v\left(t_{1}-\tau\right)>0$. On the other hand, we have

$$
v\left(t_{1}-\tau\right)=\left(\varphi_{3}(0)+N \delta \int_{0}^{t_{1}-\tau} y(u) \mathrm{e}^{\mu u} d u\right) \mathrm{e}^{-\mu\left(s_{2}-\tau\right)}=0,
$$

and this leads a contradiction.

Next, suppose that there exists a $t_{2} \geq \tau$ such that $\frac{d}{d t} v\left(t_{2}\right)>0$ and $v\left(t_{2}\right)=0$. Similarly, we obtain $y\left(t_{2}\right)>0$ from (1.3). On the other hand, we have

$$
y\left(t_{2}\right)=\left(\varphi_{2}(0)+\mathrm{e}^{-m \tau} \int_{0}^{t_{2}} f(x(s-\tau), v(s-\tau)) \mathrm{e}^{\delta s} d s\right) \mathrm{e}^{-\delta t_{2}}=0,
$$

which also leads a contradiction. Thus, the conclusion holds.

Now we define the basic reproduction number and discuss the possible equilibrium for (1.3)(1.4).

(1.3)-(1.4) always has an uninfected equilibrium $E_{0}=\left(x_{0}, 0,0\right)$, where $x_{0}=\frac{s}{d}$, corresponding to the extinction of the virus and infected cells. The basic reproduction number for (1.3)-(1.4) is given by

$$
R_{0}=\frac{k x_{0}}{1+a x_{0}} \mathrm{e}^{-m \tau} \frac{N}{\mu}
$$

We show that (1.3)-(1.4) admits a unique infected equilibrium $E_{*}=\left(x^{*}, y^{*}, v^{*}\right)$, which satisfies

$$
\left\{\begin{array}{l}
0=s-d x^{*}-f\left(x^{*}, v^{*}\right), \\
0=f\left(x^{*}, v^{*}\right) \mathrm{e}^{-m \tau}-\delta y^{*} \\
0=N \delta y^{*}-\mu v^{*}
\end{array}\right.
$$

for $R_{0}>1$. From the third equation of (2.3), we obtain $y^{*}=\frac{\mu}{N \delta} v^{*}$. By substituting this into the second equation of (2.3), we also obtain

$$
x^{*}=\frac{1+b v^{*}}{k \mathrm{e}^{-m \tau \frac{N}{\mu}-a}} .
$$

Note that the following holds

$$
\frac{k}{a} \mathrm{e}^{-m \tau} \frac{N}{\mu}>\frac{k x_{0}}{1+a x_{0}} \mathrm{e}^{-m \tau} \frac{N}{\mu}=R_{0}>1, \text { for } a>0,
$$

which implies that $k \mathrm{e}^{-m \tau} \frac{N}{\mu}-a>0$ if $R_{0}>1$. Now we show that there exists a unique positive solution $v^{*}$ which satisfies 


$$
\begin{aligned}
0 & =s-d x^{*}-f\left(x^{*}, v^{*}\right) \\
& =s-d\left(\frac{1+b v^{*}}{k \mathrm{e}^{-m \tau} \frac{N}{\mu}-a}\right)-\delta \mathrm{e}^{m \tau} y^{*} \\
& =s-d\left(\frac{1+b v^{*}}{k \mathrm{e}^{-m \tau} \frac{N}{\mu}-a}\right)-\mathrm{e}^{m \tau} \frac{\mu}{N} v^{*} .
\end{aligned}
$$

Consider the function

$$
K(v)=s-d\left(\frac{1+b v}{k \mathrm{e}^{-m \tau} \frac{N}{\mu}-a}\right)-\mathrm{e}^{m \tau} \frac{\mu}{N} v \text { for } v \in[0,+\infty) .
$$

We see that $K(v)$ is monotone decreasing on $v$. Moreover, it follows

$$
\begin{aligned}
K(0) & =s-d\left(\frac{1}{k \mathrm{e}^{-m \tau} \frac{N}{\mu}-a}\right) \\
& =\left(\frac{d}{k \mathrm{e}^{-m \tau \frac{N}{\mu}-a}}\right)\left[\left(k \mathrm{e}^{-m \tau} \frac{N}{\mu}-a\right) x_{0}-1\right] \\
& =\left(\frac{d\left(1+a x_{0}\right)}{k \mathrm{e}^{-m \tau} \frac{N}{\mu}-a}\right)\left(R_{0}-1\right)>0 .
\end{aligned}
$$

Thus, there exists a unique $v^{*}>0$ such that $K\left(v^{*}\right)=0$. Consequently, (1.3)-(1.4) admits a unique infected equilibrium

$$
E_{*}=\left(x^{*}, y^{*}, v^{*}\right)=\left(\frac{1+b v^{*}}{k \mathrm{e}^{-m \tau} \frac{N}{\mu}-a}, \frac{\mu}{N \delta} v^{*}, v^{*}\right)
$$

\section{Global stability of the uninfected equilibrium}

Theorem 3.1 If $R_{0} \leq 1$ then the uninfected equilibrium $E_{0}$ of (1.3)-(1.4) is globally asymptotically stable.

PROOF. We construct the following Lyapunov functional

$$
U(t)=\frac{x_{0}}{1+a x_{0}} \mathrm{e}^{-m \tau} U_{1}(t)+U_{2}(t)
$$

where

$$
\begin{aligned}
U_{1}(t) & =\frac{x(t)}{x_{0}}-1-\ln \frac{x(t)}{x_{0}}, \\
U_{2}(t) & =y(t)+\frac{1}{N} v(t)+\mathrm{e}^{-m \tau} \int_{t-\tau}^{t} f(x(u), v(u)) d u .
\end{aligned}
$$

We calculate the time derivative of $U(t)$ along the solutions of (1.3)-(1.4).

First, we calculate $\frac{d U_{1}(t)}{d t}$ 


$$
\begin{aligned}
\frac{d}{d t} U_{1}(t) & =\left(\frac{1}{x_{0}}-\frac{1}{x(t)}\right)(s-d x(t)-f(x(t), v(t))) \\
& =\frac{x(t)-x_{0}}{x_{0} x(t)}(s-d x(t)-f(x(t), v(t))) .
\end{aligned}
$$

Substituting $s=d x_{0}$ gives

$$
\begin{aligned}
\frac{d}{d t} U_{1}(t) & =\frac{x(t)-x_{0}}{x_{0} x(t)}\left(d x_{0}-d x(t)-f(x(t), v(t))\right) \\
& =-\frac{d}{x_{0} x(t)}\left(x(t)-x_{0}\right)^{2}-\left(\frac{1}{x_{0}}-\frac{1}{x(t)}\right) f(x(t), v(t)) .
\end{aligned}
$$

Next, we calculate $\frac{d U_{2}(t)}{d t}$

$$
\begin{aligned}
\frac{d U_{2}(t)}{d t} & =\left(f(x(t-\tau), v(t-\tau)) \mathrm{e}^{-m \tau}-\delta y(t)\right)+\left(\delta y(t)-\frac{\mu}{N} v(t)\right) \\
& +\mathrm{e}^{-m \tau}(f(x(t), v(t))-f(x(t-\tau), v(t-\tau))) \\
& =\mathrm{e}^{-m \tau} f(x(t), v(t))-\frac{\mu}{N} v(t) .
\end{aligned}
$$

Consequently, by (3.1) and (3.2), we obtain

$$
\frac{d}{d t} U(t)=-\frac{d \mathrm{e}^{-m \tau}}{\left(1+a x_{0}\right) x(t)}\left(x(t)-x_{0}\right)^{2}+C(t),
$$

where

$$
C(t)=-\frac{x_{0}}{1+a x_{0}} \mathrm{e}^{-m \tau}\left(\frac{1}{x_{0}}-\frac{1}{x(t)}\right) f(x(t), v(t))+\mathrm{e}^{-m \tau} f(x(t), v(t))-\frac{\mu}{N} v(t) .
$$

We claim $C(t)$ is not positive. Now it follows that

$$
\begin{aligned}
C(t) & =\frac{\mu}{N}\left[-\left(\frac{1}{x_{0}}-\frac{1}{x(t)}\right) \frac{R_{0}}{k} f(x(t), v(t))+\left(\frac{\mu}{N}\right)^{-1} \mathrm{e}^{-m \tau} f(x(t), v(t))-v(t)\right] \\
& =\frac{\mu}{N}\left[-\left(\frac{1}{x_{0}}-\frac{1}{x(t)}\right) \frac{R_{0}}{k} f(x(t), v(t))+R_{0}\left(\frac{k x_{0}}{1+a x_{0}}\right)^{-1} f(x(t), v(t))-v(t)\right] \\
& =\frac{\mu}{N}\left[R_{0} f(x(t), v(t)) \frac{1}{k}\left(\frac{1+a x_{0}}{x_{0}}-\frac{1}{x_{0}}+\frac{1}{x(t)}\right)-v(t)\right] \\
& =\frac{\mu}{N}\left[R_{0} f(x(t), v(t)) \frac{1}{k}\left(a+\frac{1}{x(t)}\right)-v(t)\right] \\
& =\frac{\mu}{N}\left[R_{0} \frac{k x(t) v(t)}{1+a x(t)+b v(t)}\left(\frac{1+a x(t)}{k x(t)}\right)-v(t)\right] \\
& =\frac{\mu}{N}\left[R_{0} \frac{1+a x(t)}{1+a x(t)+b v(t)} v(t)-\frac{1+a x(t)+b v(t)}{1+a x(t)+b v(t)} v(t)\right] \\
& =\frac{\mu}{N}\left[\left(R_{0}-1\right) \frac{1+a x(t)}{1+a x(t)+b v(t)} v(t)-\frac{b}{1+a x(t)+b v(t)} v(t)^{2}\right] .
\end{aligned}
$$

Since we have $R_{0} \leq 1, C(t) \leq 0$ follows. Thus, $\frac{d U(t)}{d t} \leq 0$ holds. Hence, solutions of system (1.3) with (1.4) limit to $M$, the largest invariant subset of $\left\{\frac{d U(t)}{d t}=0\right\}$. We see that $\frac{d U(t)}{d t}=0$ if and only if $x(t)=x_{0}$, and $v(t)=0$. By the above discussion, each element of $M$ satisfies $x(t)=x_{0}$, 
and $v(t)=0$ for all $t$. It follows that $y(t)=0$ for all $t$. From La Salle's invariant principle, the uninfected equilibrium $E_{0}$ is globally asymptotically stable. Thus, the proof is complete.

\section{Uniformly persistence of the solution}

In this section, by applying persistence theory (see also [2,17]), we show the uniformly persistence of the solution of (1.3)-(1.4) for $R_{0}>1$. This result shows that the virus and infected cells eventually persist for $R_{0}>1$.

Theorem 4.1 Assume that one of the initial conditions in Lemma 2.1 holds. If $R_{0}>1$, then there exists a positive constant $\varepsilon$ such that the solution $(x(t), y(t), v(t))$ of $(1.3)-(1.4)$ satisfies

$$
\varepsilon \leq \min \left\{\liminf _{t \rightarrow+\infty} y(t), \liminf _{t \rightarrow+\infty} v(t)\right\} .
$$

PROOF. In order to use persistence theory, we define $\varphi=\left(\varphi_{1}, \varphi_{2}, \varphi_{3}\right)$ and

$$
X=\left\{\varphi \in C_{+}^{3}\right\}, X_{0}=X_{0}^{1} \cup X_{0}^{2} \cup X_{0}^{3}, \partial X_{0}=X \backslash X_{0},
$$

where

$$
\begin{aligned}
& X_{0}^{1}=\left\{\varphi \in X \mid \text { there exists a } \bar{\theta} \in[-\tau, 0) \text { such that } \varphi_{1}(\theta) \varphi_{3}(\theta)>0\right\} \\
& X_{0}^{2}=\left\{\varphi \in X \mid \varphi_{2}(0)>0\right\}, \\
& X_{0}^{3}=\left\{\varphi \in X \mid \varphi_{3}(0)>0\right\} .
\end{aligned}
$$

$\partial X_{0}$ is relatively closed in $X$ and

$$
\partial X_{0}=\left\{\varphi \in X \mid \varphi_{2}(0)=\varphi_{3}(0)=\varphi_{1}(\theta) \varphi_{3}(\theta)=0 \text { for } \theta \in[-\tau, 0)\right\} .
$$

Let $\Phi(t): X \rightarrow X$ be the solution semiflow associated with (1.3)-(1.4), that is,

$$
(\Phi(t) \varphi)(\theta)=(x(t+\theta, \varphi), y(t+\theta, \varphi), v(t+\theta, \varphi)), \theta \in[-\tau, 0], t \geq 0 .
$$

From Lemmas 2.1 and 2.2, $\Phi(t): X_{0} \rightarrow X_{0}$ for any $t \geq \tau$ and $\Phi(t): \partial X_{0} \rightarrow \partial X_{0}$ for any $t \geq 0$. Furthermore, by Lemmas 2.1 and 2.2, $\Phi(t): X \rightarrow X$ is point dissipative. Set

$$
M_{\partial}=\left\{\varphi \in X \mid \Phi(t) \varphi \in \partial X_{0}, \text { for any } t \geq 0\right\} .
$$

Then, by Lemma 2.2, it follows

$$
M_{\partial}=\left\{\varphi \in \partial X_{0} \mid y(t, \varphi)=v(t, \varphi)=0 \text { for any } t \geq 0\right\} .
$$

Assume $R_{0}>1$. Then, from (2.2), it holds that

$$
\frac{k x_{0}}{1+a x_{0}} \mathrm{e}^{-m \tau}-\frac{\mu}{N}>0 \text {. }
$$

Hence, there exist a small enough $\eta>0$ and $\gamma>0$ such that

$$
\frac{f\left(x_{0}-\eta, \gamma\right)}{\gamma} \mathrm{e}^{-m \tau}-\frac{\mu}{N}=\frac{k\left(x_{0}-\eta\right)}{1+a\left(x_{0}-\eta\right)+b \gamma} \mathrm{e}^{-m \tau}-\frac{\mu}{N}>0 .
$$

Let us consider the following perturbed equation to the first equation of (1.3)

$$
\frac{d}{d t} \bar{x}(t)=s-d \bar{x}(t)-f(\bar{x}(t), v)
$$

For a fixed $v$, we put $\tilde{f}_{v}(x)=s-d x-f(x, v)$ for $x \in[0,+\infty)$. From (1.4), we see that $\tilde{f}_{v}(x)$ is monotone decreasing on $x \in[0,+\infty), \tilde{f}_{v}(0)=s-f(0, v)=s>0$ and $\lim _{x \rightarrow+\infty} \tilde{f}_{v}(x)=-\infty$. 
Therefore, (4.2) admits a unique positive equilibrium $x_{v}^{*}$ such that $\tilde{f}_{v}\left(x_{v}^{*}\right)=0$. From the above discussion, we have $\frac{d}{d t} \bar{x}(t)=\tilde{f}_{v}(\bar{x}(t))>0$ for $\bar{x}(t) \in\left[0, x_{v}^{*}\right)$ and $\frac{d}{d t} \bar{x}(t)=\tilde{f}_{v}(\bar{x}(t))<0$ for $\bar{x}(t) \in$ $\left[x_{v}^{*},+\infty\right)$ which implies that a unique positive equilibrium $x_{v}^{*}$ is globally attractive in $\mathbb{R}_{+}$. Since $x_{v}^{*}$ is continuous on $v$, we fix $v>0$ small enough such that

$$
x_{v}^{*}>x_{0}-\eta \text {. }
$$

We now show that the uninfected equilibrium $E_{0}$ repels the solutions of (1.3)-(1.4) in $X_{0}$. We claim that there exists $\gamma>0$ such that the solution semiflow $\Phi(t)$ associated with (1.3)-(1.4) satisfies

$$
\limsup _{t \rightarrow+\infty}\left\|\Phi(t) \varphi-E_{0}\right\| \geq \gamma \text { for any } \varphi \in X_{0} \text {. }
$$

Suppose that limsup $\sup _{t \rightarrow+\infty}\left\|\Phi(t) \varphi-E_{0}\right\|<\gamma$ for some $\varphi \in X_{0}$. Then there exists a $t_{1}>0$ such that $\left\|\Phi(t) \varphi-E_{0}\right\|<\gamma$ for any $t \geq t_{1}$. Set $(x(t), y(t), v(t))=\Phi(t) \varphi$. It follows that $0 \leq v(t) \leq \gamma, t \geq t_{1}$ and hence, we have that

$$
\frac{d}{d t} x(t) \geq s-d x(t)-f(x(t), \gamma), \text { for } t \geq t_{1} .
$$

We note that $f(x, v)$ is monotone increasing on $v \in[0,+\infty)$. Since the unique positive equilibrium $x_{v}^{*}$ of (4.2) is globally attractive and $x_{v}^{*}>x_{0}-\eta$, there exists a large enough $t_{2}>0$ such that

$$
x(t) \geq x_{0}-\eta \text { for } t>t_{2} .
$$

Let us consider the differential function

$$
W(t)=y(t)+\frac{1}{N} v(t)+\mathrm{e}^{-m \tau} \int_{t-\tau}^{t} f(x(u), v(u)) d u
$$

By Lemma 2.1, the function $W(t)$ is bounded above. Calculating the derivative of $W(t)$ along the solution of (1.3)-(1.4) gives

$$
\begin{aligned}
\frac{d}{d t} W(t) & =\mathrm{e}^{-m \tau} f(x(t), v(t))-\frac{\mu}{N} v(t) \\
& =\left[\frac{f(x(t), v(t))}{v(t)} \mathrm{e}^{-m \tau}-\frac{\mu}{N}\right] v(t) \\
& \geq\left[\frac{f\left(x_{0}-\eta, \gamma\right)}{\gamma} \mathrm{e}^{-m \tau}-\frac{\mu}{N}\right] v(t), \text { for } t>t_{2} .
\end{aligned}
$$

Since we have (4.1), it holds that $\lim _{t \rightarrow+\infty} W(t)=+\infty$. This leads to a contradiction to the boundedness of the function $W(t)$.

Thus (4.3) holds and $\Phi(t)$ is weakly uniformly persistent with respect to $\left(X_{0}, \partial X_{0}\right)$. Then it follows that $E_{0}$ is an isolated invariant set in $X$ and $W^{s}\left(E_{0}\right) \cap X_{0}=\emptyset$. It is clear that every solution in $M_{\partial}$ converges to $E_{0}$ and $E_{0}$ is acyclic in $M_{\partial}$. By the acyclicity theorem on uniform persistence, we obtain that $\Phi(t)$ is uniformly persistent with respect to $\left(X_{0}, \partial X_{0}\right)$ and the proof is complete.

\section{Global stability of the infected steady state}

In this section, we show that the infective equilibrium $E_{*}$ of (1.3)-(1.4) is globally asymptotically stable if $R_{0}>1$ by employing the method of a Lyapunov functional. For simplicity of the proof, we use the following notation. 


$$
\left\{\begin{array}{l}
\tilde{x}_{t}=\frac{x(t)}{x^{*}}, \tilde{y}_{t}=\frac{y(t)}{y^{*}}, \tilde{v}_{t}=\frac{v(t)}{v^{*}}, \\
\tilde{z}_{t}=\frac{f(x(t), v(t))}{f\left(x^{*}, v^{*}\right)}, \tilde{z}_{t, \tau}=\frac{f(x(t-\tau), v(t-\tau))}{f\left(x^{*}, v^{*}\right)}, \tilde{z}_{t}^{x}=\frac{f\left(x(t), v^{*}\right)}{f\left(x^{*}, v^{*}\right)},
\end{array}\right.
$$

We set

$$
g(x)=x-1-\ln x \text { for } x \in(0,+\infty) .
$$

$g(x) \geq 0$ for all $x \in(0,+\infty)$ and $\min _{0<x<+\infty} g(x)=g(1)=0$. The following lemma plays an important role in the proof of Theorem 5.2.

Lemma 5.1 Assume that one of the initial conditions in Lemma 2.1 holds. It holds that

$$
\left(\tilde{v}_{t}-\frac{\tilde{z}_{t}}{\tilde{z}_{t}^{x}}\right)\left(\frac{\tilde{z}_{t}}{\tilde{z}_{t}^{x}}-1\right) \geq 0
$$

and

$$
g\left(\tilde{v}_{t}\right)-g\left(\frac{\tilde{z}_{t}}{\tilde{z}_{t}^{x}}\right) \geq 0
$$

with equality if and only if $\tilde{v}_{t}=\frac{\tilde{t}_{t}}{\tilde{z}_{t}^{t}}=1$ for all $t \geq \tau$.

PROOF. First, we have that

$$
\begin{aligned}
\tilde{v}_{t}-\frac{\tilde{z}_{t}}{\tilde{z}_{t}^{*}} & =\frac{v(t)}{v^{*}}-\frac{f(x(t), v(t))}{f\left(x^{*}, v^{*}\right)}\left(\frac{f\left(x(t), v^{*}\right)}{f\left(x^{*}, v^{*}\right)}\right)^{-1}=\frac{v(t)}{v^{*}}-\frac{f(x(t), v(t))}{f\left(x(t), v^{*}\right)} \\
& =\frac{f(x(t), v(t))}{v^{*}}\left(\frac{v(t)}{f(x(t), v(t))}-\frac{v^{*}}{f\left(x(t), v^{*}\right)}\right),
\end{aligned}
$$

and

$$
\begin{aligned}
\frac{\tilde{z}_{t}}{\tilde{z}_{t}^{x}}-1 & =\frac{f(x(t), v(t))}{f\left(x^{*}, v^{*}\right)}\left(\frac{f\left(x(t), v^{*}\right)}{f\left(x^{*}, v^{*}\right)}\right)^{-1}-1=\frac{f(x(t), v(t))}{f\left(x(t), v^{*}\right)}-1 \\
& =\frac{f(x(t), v(t))-f\left(x(t), v^{*}\right)}{f\left(x(t), v^{*}\right)} .
\end{aligned}
$$

Then, by using (1.4), we immediately obtain

$$
\begin{aligned}
& \left(\tilde{v}_{t}-\frac{\tilde{z}_{t}}{\tilde{z}_{t}^{*}}\right)\left(\frac{\tilde{z}_{t}}{\tilde{z}_{t}^{x}}-1\right) \\
= & \frac{f(x(t), v(t))}{v^{*} f\left(x(t), v^{*}\right)}\left(\frac{v(t)}{f(x(t), v(t))}-\frac{v^{*}}{f\left(x(t), v^{*}\right)}\right)\left(f(x(t), v(t))-f\left(x(t), v^{*}\right)\right) \\
= & \frac{f(x(t), v(t))}{v^{*} f\left(x(t), v^{*}\right)}\left(\frac{1+a x(t)+b v(t)}{k x(t)}-\frac{1+a x(t)+b v^{*}}{k x(t)}\right)\left(f(x(t), v(t))-f\left(x(t), v^{*}\right)\right) \\
= & \frac{f(x(t), v(t))}{v^{*} f\left(x(t), v^{*}\right)} \frac{b\left(v(t)-v^{*}\right)}{k x(t)}\left(f(x(t), v(t))-f\left(x(t), v^{*}\right)\right) \\
\geq & 0,
\end{aligned}
$$

from which (5.1) follows. (5.1) implies that either $\tilde{v}_{t} \leq \frac{\tilde{z}_{t}}{\tilde{z}_{t}^{t}} \leq 1$ or $\tilde{v}_{t} \geq \frac{\tilde{z}_{t}}{\tilde{z}_{t}^{t}} \geq 1$ holds for all $t \geq \tau$. Thus, (5.2) holds. From the above discussion, we see that $g\left(\tilde{v}_{t}\right)=g\left(\frac{\tilde{z}_{t}}{\tilde{z}_{t}^{t}}\right)=0$ holds if and only if $\tilde{v}_{t}=\frac{\tilde{z}_{t}}{z_{t}^{t}}=1$. Hence, the proof is complete. 
We are in a position to prove the global asymptotic stability of the infected equilibrium $E_{*}$ for $R_{0}>1$.

Theorem 5.2 Assume that one of the initial conditions in Lemma 2.1 holds. If $R_{0}>1$ then the infected equilibrium $E_{*}$ of (1.3)-(1.4) is globally asymptotically stable.

PROOF. We construct the following Lyapunov functional

$$
U(t)=\frac{x^{*}}{f\left(x^{*}, v^{*}\right)} U_{x}(t)+\frac{y^{*}}{f\left(x^{*}, v^{*}\right) \mathrm{e}^{-m \tau}} U_{y}(t)+\frac{v^{*}}{N \delta y^{*}} U_{v}(t)+\bar{U}(t),
$$

where

$$
\begin{aligned}
U_{x}(t) & =\frac{x(t)}{x^{*}}-1-\frac{1}{x^{*}} \int_{x^{*}}^{x(t)} \frac{f\left(x^{*}, v^{*}\right)}{f\left(\xi, v^{*}\right)} d \xi, U_{y}(t)=g\left(\frac{y(t)}{y^{*}}\right), U_{v}(t)=g\left(\frac{v(t)}{v^{*}}\right), \\
\bar{U}(t) & =\int_{t-\tau}^{t} g\left(\frac{f(x(u), v(u))}{f\left(x^{*}, v^{*}\right)}\right) d u .
\end{aligned}
$$

Let

$$
L(x)=\frac{x}{x^{*}}-1-\frac{1}{x^{*}} \int_{x^{*}}^{x} \frac{f\left(x^{*}, v^{*}\right)}{f\left(\xi, v^{*}\right)} d \xi, \text { for } x \in(0,+\infty) .
$$

Since it holds that

$$
\frac{d}{d x} L(x)=\frac{1}{x^{*}}\left(1-\frac{f\left(x^{*}, v^{*}\right)}{f\left(x, v^{*}\right)}\right)
$$

we have that $\frac{d}{d x} L(x)<0$ for $x \in\left(0, x^{*}\right), \frac{d}{d x} L(x)>0$ for $x \in\left(x^{*},+\infty\right)$ and $\left.\frac{d}{d x} L(x)\right|_{x=x^{*}}=0$. We also have $L\left(x^{*}\right)=0$. Thus, $U_{x}(t) \geq 0$ for all $t>0$. Hence, by Lemma 2.1, we see $U(t) \geq 0$ for all $t \geq \tau$.

We now show that $\frac{d U(t)}{d t} \leq 0$. First, we calculate $\frac{d U_{x}(t)}{d t}$

$$
\begin{aligned}
\frac{d U_{x}(t)}{d t} & =\left(\frac{1}{x^{*}}-\frac{1}{x^{*}} \frac{f\left(x^{*}, v^{*}\right)}{f\left(x(t), v^{*}\right)}\right) \frac{d}{d t} x(t) \\
& =\frac{1}{x^{*}}\left(1-\frac{f\left(x^{*}, v^{*}\right)}{f\left(x(t), v^{*}\right)}\right)(s-d x(t)-f(x(t), v(t))) .
\end{aligned}
$$

Substituting $s=d x^{*}+f\left(x^{*}, v^{*}\right)$ gives

$$
\begin{aligned}
\frac{d U_{x}(t)}{d t}= & \frac{1}{x^{*}}\left(1-\frac{f\left(x^{*}, v^{*}\right)}{f\left(x(t), v^{*}\right)}\right)\left(d x^{*}+f\left(x^{*}, v^{*}\right)-d x(t)-f(x(t), v(t))\right) \\
= & \frac{1}{x^{*}}\left(1-\frac{f\left(x^{*}, v^{*}\right)}{f\left(x(t), v^{*}\right)}\right)\left(-d\left(x(t)-x^{*}\right)+f\left(x^{*}, v^{*}\right)-f(x(t), v(t))\right) \\
= & -\frac{d}{x^{*}}\left(1-\frac{f\left(x^{*}, v^{*}\right)}{f\left(x(t), v^{*}\right)}\right)\left(x(t)-x^{*}\right) \\
& \quad+\frac{f\left(x^{*}, v^{*}\right)}{x^{*}}\left(1-\frac{f\left(x^{*}, v^{*}\right)}{f\left(x(t), v^{*}\right)}\right)\left(1-\frac{f(x(t), v(t))}{f\left(x^{*}, v^{*}\right)}\right) \\
= & -\frac{d}{x^{*}}\left(1-\frac{f\left(x^{*}, v^{*}\right)}{f\left(x(t), v^{*}\right)}\right)\left(x(t)-x^{*}\right)+\frac{f\left(x^{*}, v^{*}\right)}{x^{*}}\left(1-\frac{1}{\tilde{z}_{t}^{x}}\right)\left(1-\tilde{z}_{t}\right) \\
= & -\frac{d}{x^{*}}\left(1-\frac{f\left(x^{*}, v^{*}\right)}{f\left(x(t), v^{*}\right)}\right)\left(x(t)-x^{*}\right)+\frac{f\left(x^{*}, v^{*}\right)}{x^{*}}\left(1-\frac{1}{\tilde{z}_{t}^{x}}-\tilde{z}_{t}+\frac{\tilde{z}_{t}}{\tilde{z}_{t}^{*}}\right) .
\end{aligned}
$$


We secondly calculate $\frac{d U_{y}(t)}{d t}$

$$
\begin{aligned}
\frac{d U_{y}(t)}{d t} & =\left(\frac{1}{y^{*}}-\frac{1}{y(t)}\right) \frac{d}{d t} y(t) \\
& =\frac{1}{y^{*}}\left(1-\frac{y^{*}}{y(t)}\right)\left(f(x(t-\tau), v(t-\tau)) \mathrm{e}^{-m \tau}-\delta y(t)\right) .
\end{aligned}
$$

Substituting $\delta y^{*}=f\left(x^{*}, v^{*}\right) \mathrm{e}^{-m \tau}$ gives $\delta=\frac{f\left(x^{*}, v^{*}\right) \mathrm{e}^{-m \tau}}{y^{*}}$ and

$$
\begin{aligned}
\frac{d U_{y}(t)}{d t} & =\frac{1}{y^{*}}\left(1-\frac{y^{*}}{y(t)}\right)\left(f(x(t-\tau), v(t-\tau)) \mathrm{e}^{-m \tau}-f\left(x^{*}, v^{*}\right) \mathrm{e}^{-m \tau} \frac{y(t)}{y^{*}}\right) \\
& =\frac{f\left(x^{*}, v^{*}\right) \mathrm{e}^{-m \tau}}{y^{*}}\left(1-\frac{y^{*}}{y(t)}\right)\left(\frac{f(x(t-\tau), v(t-\tau))}{f\left(x^{*}, v^{*}\right)}-\frac{y(t)}{y^{*}}\right) \\
& =\frac{f\left(x^{*}, v^{*}\right) \mathrm{e}^{-m \tau}}{y^{*}}\left(1-\frac{1}{\tilde{y}_{t}}\right)\left(\tilde{z}_{t, \tau}-\tilde{y}_{t}\right) \\
& =\frac{f\left(x^{*}, v^{*}\right) \mathrm{e}^{-m \tau}}{y^{*}}\left(\tilde{z}_{t, \tau}-\frac{\tilde{z}_{t, \tau}}{\tilde{y}_{t}}-\tilde{y}_{t}+1\right) .
\end{aligned}
$$

Let us calculate $\frac{d U_{v}(t)}{d t}$

$$
\begin{aligned}
\frac{d U_{v}(t)}{d t} & =\left(\frac{1}{v^{*}}-\frac{1}{v(t)}\right) \frac{d}{d t} v(t) \\
& =\frac{1}{v^{*}}\left(1-\frac{v^{*}}{v(t)}\right)(N \delta y(t)-\mu v(t)) .
\end{aligned}
$$

Substituting $\mu v^{*}=N \delta y^{*}$ gives $\mu=N \delta \frac{y^{*}}{v^{*}}$ and

$$
\begin{aligned}
\frac{d U_{v}(t)}{d t} & =\frac{1}{v^{*}}\left(1-\frac{v^{*}}{v(t)}\right)\left(N \delta y(t)-N \delta y^{*} \frac{v(t)}{v^{*}}\right) \\
& =\frac{N \delta y^{*}}{v^{*}}\left(1-\frac{v^{*}}{v(t)}\right)\left(\frac{y(t)}{y^{*}}-\frac{v(t)}{v^{*}}\right) \\
& =\frac{N \delta y^{*}}{v^{*}}\left(1-\frac{1}{\tilde{v}_{t}}\right)\left(\tilde{y}_{t}-\tilde{v}_{t}\right) \\
& =\frac{N \delta y^{*}}{v^{*}}\left(\tilde{y}_{t}-\tilde{v}_{t}-\frac{\tilde{y}_{t}}{\tilde{v}_{t}}+1\right) .
\end{aligned}
$$

Calculating $\frac{d \bar{U}(t)}{d t}$ gives as follows

$$
\begin{aligned}
\frac{d \bar{U}(t)}{d t} & =g\left(\frac{f(x(t), v(t))}{f\left(x^{*}, v^{*}\right)}\right)-g\left(\frac{f(x(t-\tau), v(t-\tau))}{f\left(x^{*}, v^{*}\right)}\right) \\
& =\tilde{z}_{t}-\ln \tilde{z}_{t}-\tilde{z}_{t, \tau}+\ln \tilde{z}_{t, \tau} .
\end{aligned}
$$

Consequently, by (5.4)-(5.7), we obtain

$$
\frac{d}{d t} U(t)=-\frac{d}{x^{*}}\left(1-\frac{f\left(x^{*}, v^{*}\right)}{f\left(x(t), v^{*}\right)}\right)\left(x(t)-x^{*}\right)+C(t, \tau),
$$

where 


$$
\begin{aligned}
& C(t, \tau)=\left(1-\frac{1}{\tilde{z}_{t}^{x}}-\tilde{z}_{t}+\frac{\tilde{z}_{t}}{\tilde{z}_{t}^{x}}\right)+\left(\tilde{z}_{t, \tau}-\frac{\tilde{z}_{t, \tau}}{\tilde{y}_{t}}-\tilde{y}_{t}+1\right)+\left(\tilde{y}_{t}-\tilde{v}_{t}-\frac{\tilde{y}_{t}}{\tilde{v}_{t}}+1\right) \\
& +\left(\tilde{z}_{t}-\ln \tilde{z}_{t}-\tilde{z}_{t, \tau}+\ln \tilde{z}_{t, \tau}\right) \\
& =\left(1-\frac{1}{\tilde{z}_{t}^{x}}\right)+\left(-\frac{\tilde{z}_{t, \tau}}{\tilde{y}_{t}}+1\right)+\left(-\frac{\tilde{y}_{t}}{\tilde{v}_{t}}+1\right)+\left(\frac{\tilde{z}_{t}}{\tilde{z}_{t}^{x}}-\tilde{v}_{t}\right)+\left(-\ln \tilde{z}_{t}+\ln \tilde{z}_{t, \tau}\right) \\
& =\left(1-\frac{1}{\tilde{z}_{t}^{x}}\right)+\left(-\frac{\tilde{z}_{t, \tau}}{\tilde{y}_{t}}+1\right)+\left(-\frac{\tilde{y}_{t}}{\tilde{v}_{t}}+1\right)+\left(\frac{\tilde{z}_{t}}{\tilde{z}_{t}^{x}}-\tilde{v}_{t}\right) \\
& +\left(\ln \frac{1}{\tilde{z}_{t}^{x}}-\ln \frac{1}{\tilde{z}_{t}^{x}}\right)+\left(\ln \frac{\tilde{z}_{t, \tau}}{\tilde{y}_{t}}-\ln \tilde{z}_{t}\right)+\left(\ln \frac{\tilde{y}_{t}}{\tilde{v}_{t}}+\ln v_{t}\right) \\
& =\left(1-\frac{1}{\tilde{z}_{t}^{x}}+\ln \frac{1}{\tilde{z}_{t}^{x}}\right)+\left(-\frac{\tilde{z}_{t, \tau}}{\tilde{y}_{t}}+1+\ln \frac{\tilde{z}_{t, \tau}}{\tilde{y}_{t}}\right)+\left(-\frac{\tilde{y}_{t}}{\tilde{v}_{t}}+1+\ln \frac{\tilde{y}_{t}}{\tilde{v}_{t}}\right) \\
& +\left(\frac{\tilde{z}_{t}}{\tilde{z}_{t}^{x}}-\tilde{v}_{t}\right)+\left(-\ln \frac{1}{\tilde{z}_{t}^{x}}\right)+\left(-\ln \tilde{z}_{t}\right)+\ln \tilde{v}_{t} \\
& =-\left[g\left(\frac{1}{\tilde{z}_{t}^{x}}\right)+g\left(\frac{\tilde{z}_{t, \tau}}{\tilde{y}_{t}}\right)+g\left(\frac{\tilde{y}_{t}}{\tilde{v}_{t}}\right)\right]+\left[\left(\frac{\tilde{z}_{t}}{\tilde{z}_{t}^{x}}-\ln \frac{\tilde{z}_{t}}{\tilde{z}_{t}^{x}}\right)-\left(\tilde{v}_{t}-\ln \tilde{v}_{t}\right)\right] \\
& =-\left[g\left(\frac{1}{\tilde{z}_{t}^{x}}\right)+g\left(\frac{\tilde{z}_{t, \tau}}{\tilde{y}_{t}}\right)+g\left(\frac{\tilde{y}_{t}}{\tilde{v}_{t}}\right)\right]-\left[g\left(\tilde{v}_{t}\right)-g\left(\frac{\tilde{z}_{t}}{\tilde{z}_{t}^{x}}\right)\right] .
\end{aligned}
$$

From (1.4), it holds that

$$
\left(1-\frac{f\left(x^{*}, v^{*}\right)}{f\left(x(t), v^{*}\right)}\right)\left(x(t)-x^{*}\right) \geq 0 .
$$

From (5.8) and Lemma 5.1, we also see $C(t, \tau) \leq 0$. Consequently, $\frac{d U(t)}{d t} \leq 0$ holds. Hence, the solution of system (1.3)-(1.4) limit to $M$, the largest invariant subset of $\left\{\frac{d U(t)}{d t}=0\right\}$.

Next we show that $M$ consists of only the infected steady state $E_{*}$. We recall that by Lemma $2.1, \frac{d U(t)}{d t}=0$ if and only if $\tilde{z}_{t}^{x}=1, \frac{\tilde{z}_{t, \tau}}{\tilde{y}_{t}}=1$, $\frac{\tilde{y}_{t}}{\tilde{v}_{t}}=1$ and $\tilde{v}_{t}=\frac{\tilde{z}_{t}}{\tilde{z}_{t}^{t}}=1$, or equivalently, if and only if $x(t)=x^{*}, y(t)=y^{*}$ and $v(t)=v^{*}$. By the above discussion, each element of $M$ satisfies $x(t)=x^{*}$, $y(t)=y^{*}$ and $v(t)=v^{*}$ for all $t \geq \tau$. It follows from Theorem 4.1 and La Salle's invariant principle that infected steady state $E_{*}$ is globally asymptotically stable. This completes the proof.

\section{Applications}

Li and Ma [8] proposed the following HIV-1 infection model with delay and nonlinear incidence rate

$$
\left\{\begin{array}{l}
\frac{d}{d t} x(t)=s-d x(t)-\frac{k x(t) v(t)}{1+v(t)}, \\
\frac{d}{d t} y(t)=\frac{k x(t-\tau) v(t-\tau)}{1+v(t-\tau)}-\delta y(t), \\
\frac{d}{d t} v(t)=N \delta y(t)-\mu v(t) .
\end{array}\right.
$$

(6.1) has a unique infected equilibrium $E_{*}=\left(x^{*}, y^{*}, v^{*}\right)$ if

$$
R_{0(6.1)}=k x_{0} \frac{N}{\mu}>1 \text {. }
$$


They showed that the infected equilibrium $E_{*}$ is locally asymptotically stable if $R_{0(6.1)}>1$ and conjectured that the infected equilibrium $E_{*}$ is globally asymptotically stable if $R_{0(6.1)}>1$. From Theorem 5.2, we obtain an affirmative answer for the problem.

Corollary 6.1 Assume that one of the initial conditions in Lemma 2.1 holds. If $R_{0(6.1)}>1$ then the infected equilibrium $E_{*}$ of (6.1) is globally asymptotically stable.

We also obtain the following viral infection model with bilinear incidence rate from (1.3)-(1.4) with $a=b=0$.

$$
\left\{\begin{array}{l}
\frac{d}{d t} x(t)=s-d x(t)-k x(t) v(t), \\
\frac{d}{d t} y(t)=k \mathrm{e}^{-m \tau} x(t-\tau) v(t-\tau)-\delta y(t), \\
\frac{d}{d t} v(t)=N \delta y(t)-\mu v(t) .
\end{array}\right.
$$

(6.2) always has an uninfected equilibrium $E_{0}$. On the other hand, there exists a unique infected equilibrium $E^{*}=\left(x^{*}, y^{*}, v^{*}\right)$ if

$$
R_{0(6.2)}=k x_{0} \mathrm{e}^{-m \tau} \frac{N}{\mu}>1
$$

The following result is derived from Theorems 3.1 and 5.2.

Corollary 6.2 i) If $R_{0(6.2)} \leq 1$ then the uninfected equilibrium $E_{0}$ of (6.2) is globally asymptotically stable.

ii) Assume that one of the initial conditions in Lemma 2.1 holds. If $R_{0(6.2)}>1$ then the infected equilibrium $E_{*}$ of (6.2) is globally asymptotically stable.

Li and Shu [9] also obtained this threshold type result by using the method of Lyapunov functional.

\section{Discussion}

Many authors have investigated the global dynamics of viral infection models. Korobeinikov [5] studied the basic viral infection model (1.1) with using Lyapunov functions. It is shown that the basic reproduction number $R_{0(1.1)}$ is an important parameter which determines the global dynamics of the model. Huang et al. [4] considered a general viral infection model (1.2) which has Beddington-DeAngelis response as a incidence rate of the virus infection. They also showed that the basic reproduction number $R_{0(1.2)}$ works as an important parameter which determines the equilibrium that is globally asymptotically stable. Recently, the global dynamics for a delayed viral infection model which has bilinear incidence rate was analyzed by Li and Shu [9].

In this paper, following Huang et al. [4], we also assume that the incidence rate of the virus infection is described by Beddington-DeAngelis response. Then we obtain the viral infection model with a latent period by introducing a discrete time delay. We investigate its global dynamics by using two Lyapunov functional and persistence theory. These Lyapunov functionals are motivated by the works by Huang et al. [4], Korobeinikov [5] and McCluskey [10].

We show that the uninfected equilibrium $E_{0}$ is globally asymptotically stable if $R_{0} \leq 1$. Moreover, the virus and infected cells eventually persist and there exists a unique infected equilibrium $E_{*}$ which is globally asymptotically stable if $R_{0}>1$. The basic reproduction number $R_{0}$ determines the equilibrium that is globally asymptotically stable, even if there is a latent period for the cell infection. 
In Section 6, we obtain an affirmative answer for the conjecture by Li and Ma [8]. The infected equilibrium of their model (6.1) is also globally asymptotically stable if it exists. We see that such a latent period in the infection does not change the virus dynamics dramatically when compared with models with no delays. The latent period in the infection does not produce a periodic solution. The basic reproduction number determines the equilibrium that is globally asymptotically stable, even if there exists a time delay in the cell infection.

\section{Acknowledgements}

The author is very grateful to the anonymous referee for carefully reading and valuable comments which leads to a significant improvement of the original manuscript. This work was partially done during the author's stay as a member of International Research Training Group (IGK 1529) at the Technical University Darmstadt.

\section{References}

[1] S. Bonhoeffer, R. M. May, G. M. Shaw, M. A. Nowak, Virus dynamics and drug therapy, Proceedings of the National Academy of Sciences of the United States of America 94 (13) (1997) 6971-6976.

[2] J. Hale, P. Waltman, Persistence in infinite-dimensional systems, SIAM Journal on Mathematical Analysis 20 (1989) 388.

[3] A. Herz, S. Bonhoeffer, R. Anderson, R. May, M. Nowak, Viral dynamics in vivo: limitations on estimates of intracellular delay and virus decay, Proceedings of the National Academy of Sciences 93 (14) (1996) 7247-7251.

[4] G. Huang, W. Ma, Y. Takeuchi, Global properties for virus dynamics model with beddington-deangelis functional response, Applied Mathematics Letters DOI: 10.1016/j.aml.2009.06.004 (2009) -.

[5] A. Korobeinikov, Global properties of basic virus dynamics models, Bull. Math. Biol. 66 (4) (2004) 879-883.

[6] A. Korobeinikov, Global properties of infectious disease models with nonlinear incidence, Bull. Math. Biol. 69 (6) (2007) 1871-1886.

[7] A. Korobeinikov, Global asymptotic properties of virus dynamics models with dose-dependent parasite reproduction and virulence and non-linear incidence rate, Math Med Biol DOI:10.1093/imammb/dqp006.

[8] D. Li, W. Ma, Asymptotic properties of a HIV-1 infection model with time delay, J. Math. Anal. Appl. 335 (1) (2007) 683-691.

[9] M. Y. Li, H. Shu, Global dynamics of an in-host viral model with intracellular delay, Bulletin of Mathematical Biology DOI:10.1007/s11538-010-9503-x.

[10] C. C. McCluskey, Complete global stability for an SIR epidemic model with delay - distributed or discrete, Nonlinear Analysis: Real World Applications 11 (1) (2010) 55 - 59.

[11] J. E. Mittler, B. Sulzer, A. U. Neumann, A. S. Perelson, Influence of delayed viral production on viral dynamics in hiv-1 infected patients, Mathematical Biosciences 152 (2) (1998) 143 - 163.

[12] P. W. Nelson, A. S. Perelson, Mathematical analysis of delay differential equation models of HIV-1 infection, Math. Biosci. 179 (1) (2002) 73-94.

[13] M. Nowak, C. Bangham, Population dynamics of immune responses to persistent viruses, Science 272 (5258) (1996) 74.

[14] A. S. Perelson, P. W. Nelson, Mathematical analysis of hiv-1 dynamics in vivo, SIAM Review 41 (1) (1999) 3-44.

[15] T. Revilla, G. Garcia-Ramos, Fighting a virus with a virus: a dynamic model for hiv-1 therapy, Mathematical Biosciences 185 (2) (2003) $191-203$.

[16] X. Song, A. U. Neumann, Global stability and periodic solution of the viral dynamics, Journal of Mathematical Analysis and Applications 329 (1) (2007) 281 - 297.

[17] X.-Q. Zhao, Dynamical systems in population biology, CMS Books in Mathematics/Ouvrages de Mathématiques de la SMC, 16, Springer-Verlag, New York, 2003.

[18] H. Zhu, X. Zou, Impact of delays in cell infection and virus production on HIV-1 dynamics, Mathematical Medicine and Biology 25 (2) (2008) 99.

[19] H. Zhu, X. Zou, Dynamics of a HIV-1 Infection model with cell-mediated immune response and intracellular delay, Discrete Contin. Dyn. Syst. Ser. B 12 (2) (2009) 511-524. 